\title{
Controle e previsão de níveis de ruído e vibrações gerados pelo desmonte de rochas com explosivos
}

\author{
Control and prediction of noise and ground vibrations generated by rock blasting \\ Felipe Tavares Dorneles', Luis Eduardo de Souza², Raul Oliveira Neto² \\ 'Programa de Pós-Graduação em Tecnologia Mineral, Universidade Federal do Pampa, Caçapava do Sul, Brasil \\ ${ }^{2}$ Doutores, Programa de Pós-Graduação em Tecnologia Mineral, Universidade Federal do Pampa, Campus de Caçapava do Sul, Brasil
}

\begin{abstract}
Resumo
De maneira geral, os crescimentos econômico e populacional aumentam a demanda por recursos minerais e, a cada ano, podemos observar pedreiras e mineradoras cada vez maiores e mais próximas às aglomerações urbanas, de modo que seus efeitos sobre as populações são mais notados. Dentre os efeitos das atividades mineiras sobre o meio ambiente, os oriundos do impacto do uso de explosivos são geralmente mais lembrados pela opinião pública, sendo que numa operação de desmonte com explosivos típica, cerca de 20-30\% da quantidade total de energia do explosivo é utilizada na fragmentação de rocha, enquanto que o restante é perdido na forma de vibrações no terreno, ruído e calor. Desta forma, há um risco real associado com esta operação, tanto para seres humanos quanto para o meio-ambiente. Além disso, pela sensibilidade humana às vibrações e ao ruído, sempre que uma operação de desmonte é realizada próxima a uma área urbana, reclamações da comunidade são uma constante, mesmo que os níveis destes impactos estejam abaixo dos limites exigidos pela legislação. Neste sentido, este trabalho está inserido no desenvolvimento de um programa de monitoramento dos níveis de vibração e ruído provocados por desmonte com explosivo, nas várias cavas de calcário atualmente explotadas no município de Caçapava do Sul, com o objetivo de propor um modelo matemático para a atenuação destas vibrações. A partir da obtenção destas equações de atenuação, espera-se elaborar um zoneamento das cargas máximas por espera a serem praticadas nas áreas em estudo, proporcionando melhorias na rotina das empresas no que se refere à fragmentação, além de pautar os níveis de vibração e ruído nos limites considerados toleráveis pela legislação atual.
\end{abstract}

Palavras-chaves: Desmonte de rochas, Explosivos, Impactos ambientais, Ruídos, Vibração.

\begin{abstract}
Air blast and ground vibrations generated by rock blasting have become a major legal and technical problem, mainly due the growing of the cities to the neighborhood of mines and quarries. For many people, the main environmental impacts associated with mining are those generated by rock blasting, and there is a real risk in handling explosives since just around $20-30 \%$ of the explosives energy is used to fragment and throw the rock. But the effects of explosives can be precalculated and predicted quite accurately, according the regulations for the use of explosives which are meant to be such a safeguard against accidents. These rules or laws establish that it is necessary to carry out a risk analysis in order to determine, first, what size vibrations the environment will accept and second, how large a charge can be blasted at certain distance without exceeding that vibration or noise limits. This risk analysis has been made in the limestone mines of Caçapava do Sul involving a careful examination of the factors that can affect the blasting operations. At the same time, a mathematical model has been developed in order to reliably predict the noise and vibration levels for a determined distance in the vicinity of the blasting place.
\end{abstract}

Keywords: Rock blasting; Explosives; Environmental impacts; Noise; Ground vibrations. 


\section{INTRODUÇÃO}

Com o crescimento econômico e, consequentemente, com o incremento do número de obras de infraestrutura observados nas últimas décadas, tem sido possível notar um aumento significativo na demanda por bens minerais e agregados para construção civil. Ao contrário de outras atividades industriais, na mineração a definição do local para estabelecimento de uma mina é condicionada pela existência e localização do minério, não seguindo apenas fatores de mercado, mas principalmente a geologia, o que acaba muitas vezes fazendo com que algum empreendimento de mineração tenha que estabelecer-se próximo de zonas urbanas, de estruturas ou obras civis e mesmo de áreas de proteção ambiental. Da mesma forma, o baixo valor agregado relacionado aos materiais para construção civil, onde o custo do frete tem potencial para afetar significativamente o preço final do produto, faz com que se busquem alternativas de extração próximas dos centros consumidores (cidades).

Desta maneira, é possível observar um crescente estabelecimento de empreendimentos de mineração no entorno de cidades, assim como aglomerações urbanas estabelecidas na vizinhança de minas e/ou pedreiras, muitas vezes sem qualquer política de ordenamento ou planejamento urbanístico, com loteamentos clandestinos ou, em algumas situações, autorizados pelo poder público, descumprindo os planos diretores dos municípios, quando estes existem (Munaretti et al., 2006a e 2006b; Koppe et al., 2001).

As atividades de mineração são potenciais fontes de perturbação para a população e o meio ambiente. Dentre os inúmeros efeitos gerados pela mineração, os oriundos do uso de explosivos são os mais lembrados pela opinião pública e, neste sentido, há a necessidade de controlarem-se os efeitos que podem causar danos às propriedades, equipamentos e instalações, além de risco à saúde e vida de trabalhadores e pessoas que residem próximas às áreas de mineração (Dallora Neto \& Ferreira, 2006; Giraudi et al., 2009; Gama, 2008; Siskind \& Stagg, 1985).

Neste sentido, este trabalho apresenta um estudo de caso onde foi realizada uma campanha de monitoramento dos níveis de ruído e vibração provocados pela operação de desmonte em uma das maiores mineradoras de calcário do município de Caçapava do Sul. A partir do levantamento destas informações, foram criados modelos matemáticos de atenuação, capazes de auxiliar na previsão dos níveis de vibração e ruídos praticados, além de permitir a otimização do desmonte, com a elaboração de mapas de distribuição de cargas máximas, de acordo com os limites estabelecidos pela legislação atual.

\subsection{Contexto Geológico}

A área de estudo encontra-se inserida no Complexo Metamórfico Vacacaí (em maior abrangência), localmente caracterizado por anfibolitos, mica-xisto, talco-xisto e lentes de mármore, com também parte da área abrangendo a Suíte Granítica Caçapava do Sul (Figura 1). Nesta região é realizada a extração de calcário metamorfizado (do tipo marga) ou mármore, inserido na unidade metavulcanossedimentar do Complexo, uma rocha metamórfica que possui entre $50 \%$ e $95 \%$ de carbonatos formados a partir de calcários e dolomitos submetidos a pressões e temperaturas elevadas (Bortolotto, 1987). O depósito, de maneira geral, possui uma forma lenticular com direção preferencial Norte-Sul, mergulhando em média $30^{\circ}$ para leste. Entre as camadas (ou bandamentos) ocorrem intrusões graníticas supergênicas, em sentido concordante em relação ao calcário e, em algumas partes, em sentido discordante. Observam-se também intrusões de diabásio (formado posteriormente ao granito), intrudidos em sentido discordante àquele do calcário. $\mathrm{O}$ material pode apresentar-se tanto de forma calcítica $\left[\mathrm{CaCO}_{3}\right]$ ou dolomítica $\left[\mathrm{CaMg}\left(\mathrm{CO}_{3}\right)^{2}\right]$. De acordo com a mineralogia e, consequentemente, sua composição química, diferentes destinações são dadas ao minério: enquanto o material calcítico, após passar por processo de calcinação, é empregado na fabricação de cal e argamassa, o calcário dolomítico, em função das características químicas, é empregado como corretivo de solo.

Em função desta complexidade geológica, com grande presença de intrusões e alto grau de fraturamento, não é possível a utilização destas rochas para fins ornamentais e de revestimento.

\section{MONITORAMENTO SÍSMICO}

Foram acompanhados dezessete eventos de detonação, em uma campanha de monitoramento realizada no período de fevereiro a abril de 2013, utilizando-se microssismógrafos de engenharia 


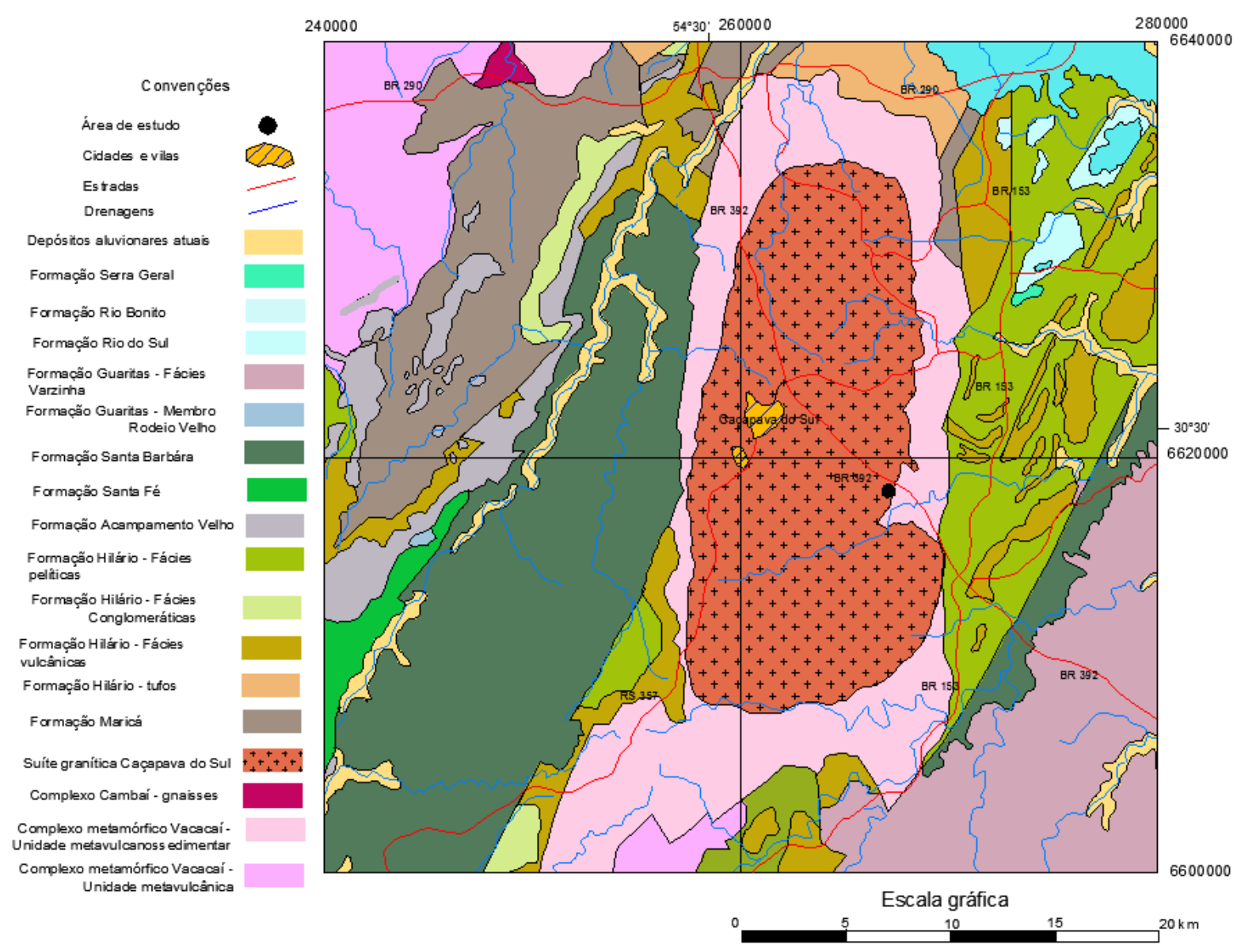

Figura 01 - Mapa geológico regional simplificado (modificado de CPRM, 2000)

portáteis Geosonics ${ }^{\circledR}$, obtendo-se trinta medições referentes aos níveis de vibração e ruídos, sendo que os pontos de monitoramento foram escolhidos de maneira a mapear estes níveis, para diferentes distâncias entre os pontos de medição e os locais de detonação, nas áreas de entorno das minas objeto deste estudo (Figura 02).

De maneira geral, foi utilizada como carga de coluna o ANFO (Nitrato de Amônia+óleo combustível) e, como carga de fundo, emulsão. Em todos os eventos de detonação, as amarrações em superfície e a iniciação dos explosivos eram feitas por meio de cordel detonante, promovendo uma iniciação lateral das cargas. Pode se observar, no entanto, que não havia uma homogeneidade nas cargas, com as mesmas sendo decididas no instante do carregamento, de acordo com a situação dos furos, muitas vezes utilizando explosivos encartuchados ou à granel nos mesmos furos e sem controle rigoroso das quantidades (massas) empregadas.

Neste sentido, para efeito da obtenção das cargas máximas por espera e do cálculo das razões de carga, utilizou-se as informações disponibilizadas pelo corpo técnico da Empresa, contendo dados como: número de furos total e por espera, profundidade, espaçamento e afastamento entre furos, tipo e densidade dos explosivos utilizados, comprimento de tampão e tempos de retardo.

\subsection{Análise dos Dados Obtidos}

Os dados resultantes da campanha de monitoramento foram analisados no software AnalysisNET, que acompanha os sismógrafos Geosonics ${ }^{\circledR}$ e que apresenta, para cada evento monitorado, o comportamento das ondas de vibração ao longo de três eixos ortogonais, além da sobrepressão acústica, durante o intervalo de medição (Figura 03). Desta forma, é possível obter-se os valores de velocidade de pico de partícula e suas respectivas frequências, de acordo com o que estabelece a NBR-9653 (ABNT, 2005), que define valores máximos permissíveis de velocidade de partícula para diferentes faixas de frequência (Figura 04). 


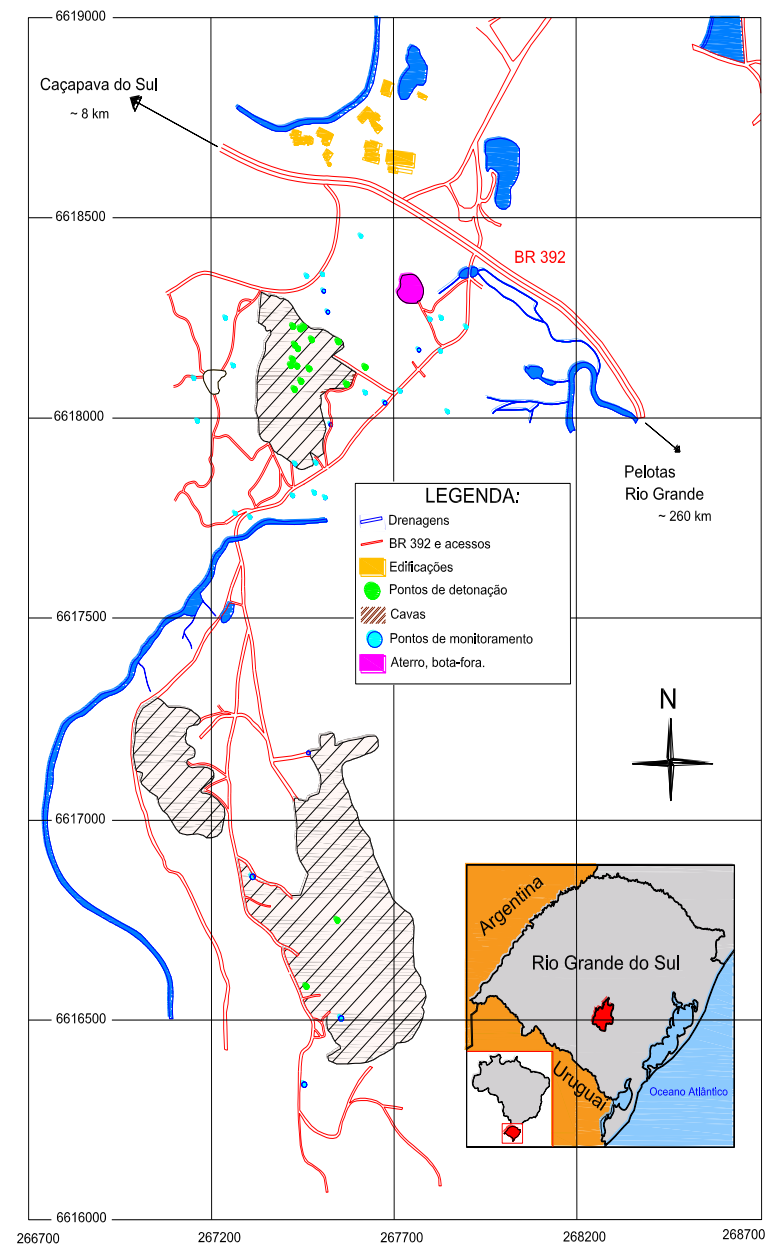

Figura 02 - Mapa de localização da área de estudo com detalhe da posição dos pontos de detonação e das estações de monitoramento

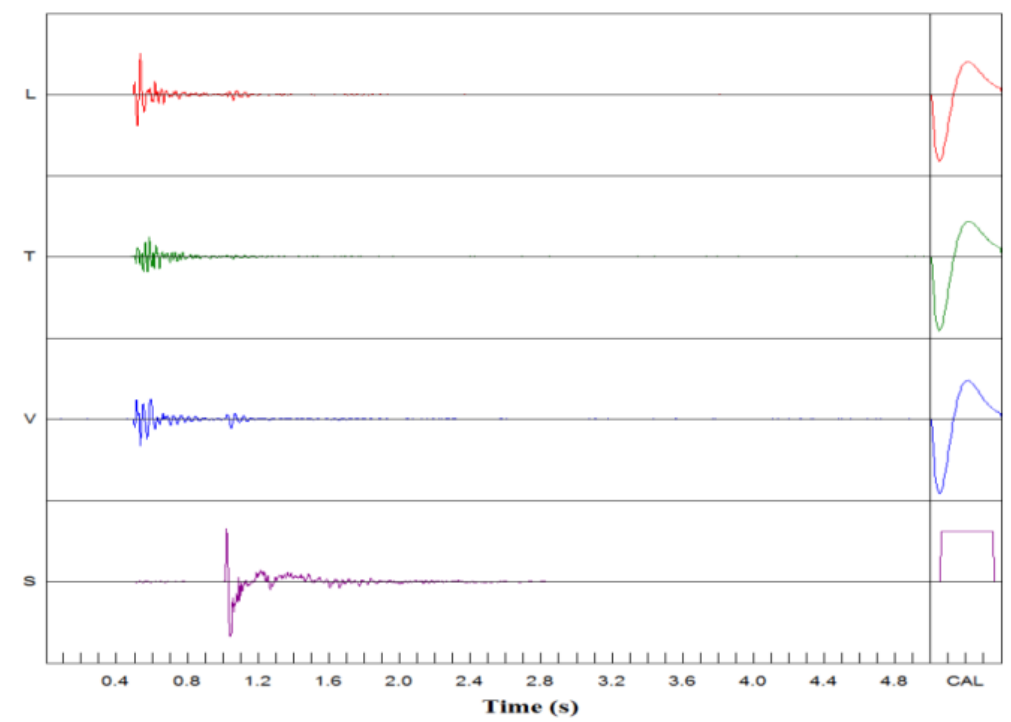

Figura 03 - Exemplo do registro gráfico de um dos eventos monitorados, com o comportamento da vibração ao longo de suas três componentes ortogonais (L: eixo longitudinal, T: transversal e V: vertical) e ruído (S) 


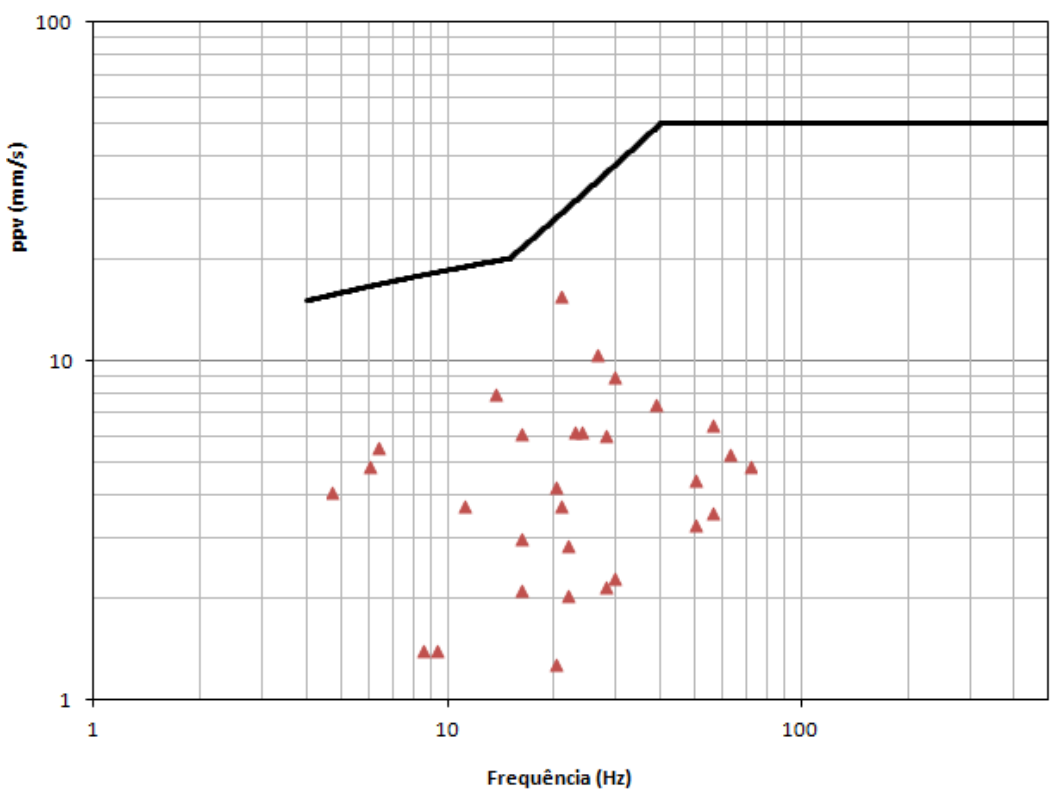

Figura 04 - Resultados dos eventos monitorados em termos de velocidade de pico de partícula (ppv, em mm/s) e suas respectivas frequências. A linha contínua representa os valores máximos permissíveis de acordo com a NBR-9653 (ABNT, 2005)

Conforme se pode observar nos resultados apresentados na Figura 04, nenhum dos eventos monitorados ultrapassou os limites definidos pelas normas no que se refere aos níveis de vibração, com uma maior concentração de valores de velocidade de pico de partícula ocorrendo entre 2 e $5 \mathrm{~mm} / \mathrm{s}$, ou seja, não implicando em risco de ocorrência de danos.

Já os níveis de ruído se mantiveram elevados em quase todos os eventos, com 06 deles excedendo o estabelecido pelas normas, ou seja, com valores acima de $134 \mathrm{~dB}$ (Figura 05). As razões estão provavelmente associadas com a expulsão do tampão pela iniciação lateral promovida pelo cordel e a utilização de material inadequado como tampão, com a consequente ejeção dos gases resultantes da reação de detonação, além do ruído provocado pela própria detonação do cordel utilizado nas ligações dos furos em superfície.

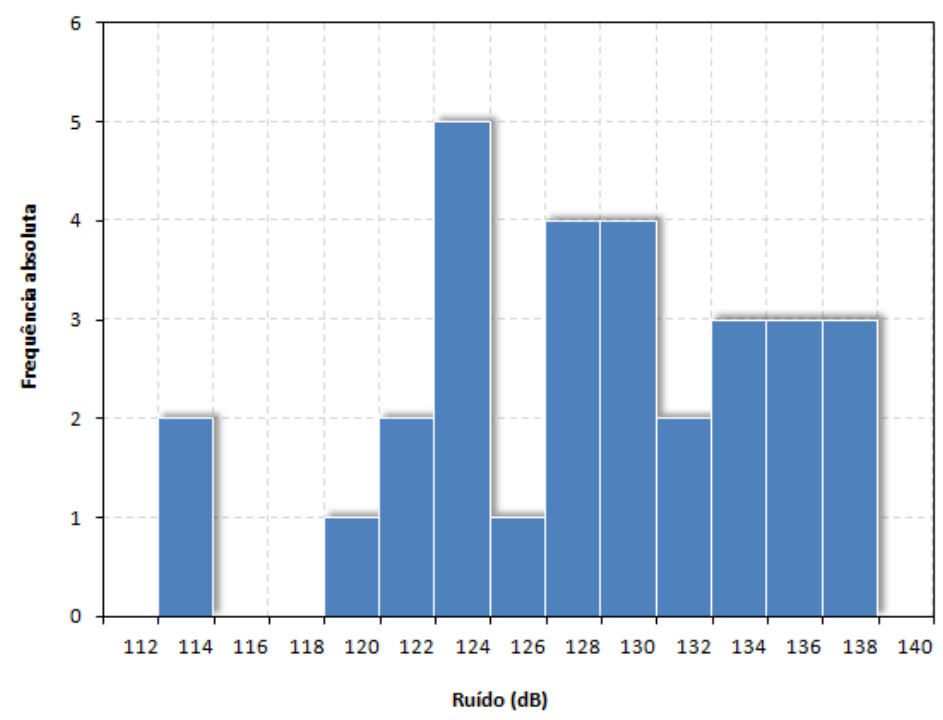

Figura 05 - Distribuição de frequência dos níveis de ruído monitorados durante os eventos de detonação acompanhados 


\section{MODELO DE ATENUAÇÃO}

Mesmo sabendo que a vibração diminui com a distância, como um maciço rochoso não é um meio isotrópico, nem sempre é simples prever o nível das vibrações para certas distâncias, pois diferentes tipos de ondas irão se propagar de forma e com velocidades diferentes, além de serem afetadas pelos seguintes fatores:

(i) presença de acamadamento, xistosidade e foliação, além de ângulo de mergulho e direção principal das camadas;

(ii) grau de fraturamento, presença de planos de fraqueza e material de preenchimento;

(iii) variações nos níveis do lençol freático;

(iv) frequência.

Além disso, a geometria do desmonte, o desempenho do explosivo, o grau de acoplamento entre explosivo e rocha e a distribuição dos intervalos de iniciação também afetam diretamente o nível de vibração.

Diversos trabalhos experimentais têm sido realizados ao longo do tempo com o objetivo de relacionar matematicamente o nível de vibração, tamanho de carga e distância. Neste sentido, costuma ser adotada a seguinte relação empírica:

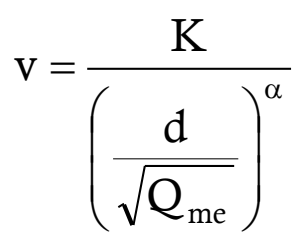

onde: $\quad \mathrm{v}=$ velocidade de partícula prevista $(\mathrm{mm} / \mathrm{s})$;

Qme = carga máxima de explosivo por espera $(\mathrm{kg})$;

$\mathrm{d}=$ distância do desmonte $(\mathrm{m})$;

$\mathrm{K}$ e $\alpha=$ constantes que dependem da geometria do desmonte, do tipo de explosivo, do tipo e estado do maciço, etc.

Assim, para aplicar a equação e predizer com adequado grau de confiança os níveis de vibração para uma determinada distância, é necessária a realização de testes, com desmontes na área de interesse ou em sua vizinhança imediata, para definição dos valores das constantes $\mathrm{K} \mathrm{e} \alpha$. Nestes testes, os dados referentes às distâncias escalonadas ( $\mathrm{DE}=\mathrm{d} / \sqrt{\mathrm{Q}_{\mathrm{me}}}$ ) são relacionados com as velocidades de pico das partículas, medidas por sismógrafos, em diagramas log-log, conforme ilustrado na Figura 06. A partir dos valores medidos, são obtidos por regressão linear os valores das constantes e, consequentemente, da equação de atenuação para a área de interesse.

Para análises de risco associado ao desmonte, velocidades de pico de partículas máximas podem ser associadas com danos em estruturas e, assim, utilizadas para definição da carga máxima a ser utilizada. No entanto, é importante ter em mente que mesmo para estas cargas, a velocidade máxima de partícula ainda pode ser excedida, já que é resultado de um ajuste matemático que depende da quantidade e correção das informações disponíveis. Assim, é recomendável adicionar um fator de segurança aos critérios de dano (reduzindo a carga máxima) ou utilizando uma reta com um limite de confiança superior daquele indicado pela reta de regressão. Além disso, cabe salientar que as cargas de ANFO, emulsão ou blendados podem resultar em diferentes velocidades de partícula, devido as diferenças nas suas velocidades de detonação e geração de onda de choque.

\subsection{INTERVALOS DE CONFIANÇA DAS EQUAÇÕES DE ATENUAÇÃo}

Quando velocidades de pico de partícula para diferentes distâncias escalonadas são lançadas em gráficos é comum observar-se um espalhamento considerável em relação à reta de regressão, que representa um modelo mediano (50\%) obtido por ajuste de mínimos quadrados a partir dos valores logarítmicos. Diversos fatores contribuem para este espalhamento, podendo-se ressaltar: geologia, condições de confinamento, tipo de explosivos, propriedades da rocha, ocorrência de fenômenos de 
a)

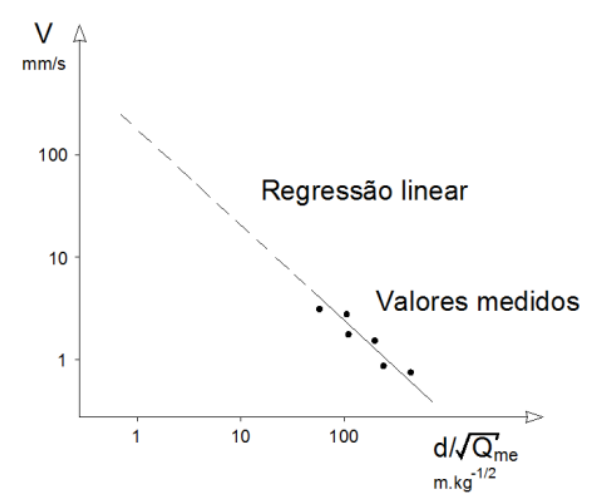

b)

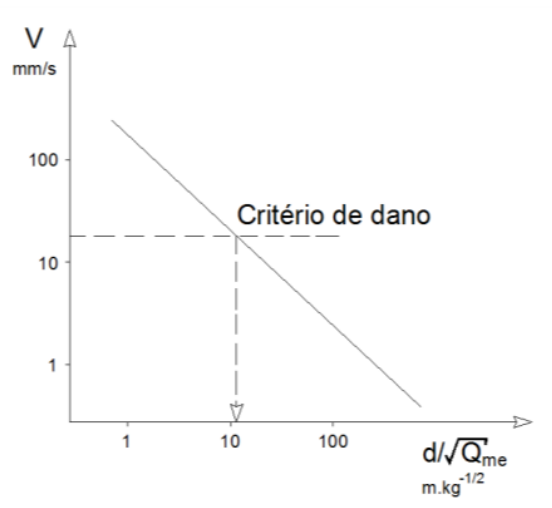

Figura 06 - Em (a), obtenção da equação de atenuação em diagramas log-log e, em (b), a determinação do valor mínimo de $d / \sqrt{\mathrm{Q}_{\mathrm{m}}}$ a ser utilizado, para a máxima velocidade de partícula que não implique em dano.

cargas cooperativas, tempos de retardo, interações entre os diferentes tipos de ondas, além de erros de medida e análise.

Conforme Larson \& Ferber (2010), o grau ou nível de confiança é a probabilidade de que o intervalo estimado contenha o parâmetro populacional, ou seja, um nível em que todos ou a maior parte dos dados estejam abaixo ou dentro do intervalo estabelecido pelo mesmo. Assim, um intervalo de confiança de $90 \%$ nos permitiria assumir que em $90 \%$ dos eventos de detonações, a velocidade de partícula não ultrapassa os limites estabelecidos.

A Figura 07 apresenta os modelos de previsão para os níveis de confiança de 84,1\% e 97,9\%, com suas respectivas equações de atenuação. Além disso, para efeito de comparação, plotou-se o modelo para velocidade resultante de partícula (VR), determinada pela soma vetorial da maior velocidade de partícula de cada componente, independentemente do instante em que ocorreram.

Assim, pode-se optar entre os modelos, aquele que fornece o nível de confiança mais de acordo para cada situação de exposição ao risco. Por exemplo, definindo-se como limite de vibração máxima a velocidade de partícula de $8 \mathrm{~mm} / \mathrm{s}$, pode-se determinar cargas máximas por espera para os diferentes níveis de confiança. De acordo com a Tabela 01 , se pode perceber que os valores de carga máxima são menores, de acordo com o incremento do nível de confiança adotado, mantendo-se fixa a distância do

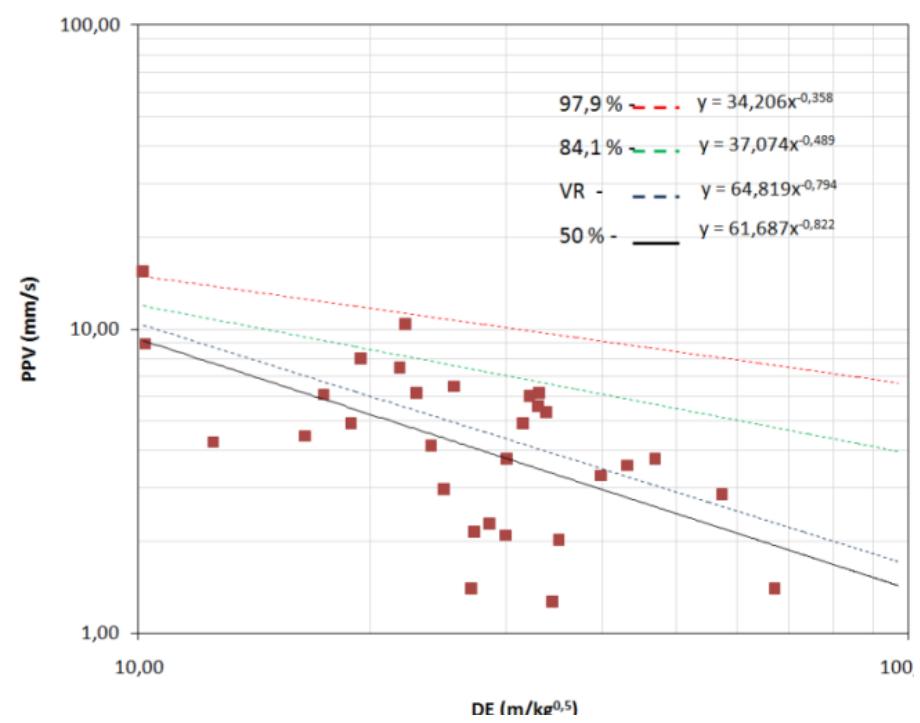

Figura 07 - Modelos de regressão entre velocidade de pico de partícula e distância escalonada, para diferentes intervalos de confiança 
local de detonação em relação ao ponto de interesse, no caso $150 \mathrm{~m}$.

Tabela 01 - Valores de carga máxima por espera para as equações de atenuação com diferentes níveis de confiança

\begin{tabular}{cc}
\hline $\begin{array}{c}\text { Nível de confiança } \\
(\%)\end{array}$ & $\begin{array}{c}\text { Carga máxima por } \\
\text { espera }(\mathrm{kg})\end{array}$ \\
\hline 50,0 & 156,215 \\
84,1 & 42,819 \\
97,9 & 6,713 \\
\hline
\end{tabular}

Da mesma forma, se fizermos a opção por um determinado modelo de atenuação, por exemplo aquele que traduza a maior segurança na definição da carga máxima, ou seja, com intervalo de confiança de 97,9\%, podemos estabelecer um zoneamento para as cargas, estimando seu valor máximo para diferentes distâncias do ponto de detonação e assumindo um nível máximo de vibração aceitável (Tabela 02).

Tabela 02 - Cargas máximas por espera calculadas para diferentes distâncias, para o modelo de atenuação com intervalo de confiança de 97,9\%, utilizando um nível máximo de vibração aceitável de $15 \mathrm{~mm} / \mathrm{s}$

\begin{tabular}{cc}
\hline $\begin{array}{c}\text { Distância ou Raio } \\
(\mathrm{m})\end{array}$ & $\begin{array}{c}\text { Carga máxima por } \\
\text { espera }(\mathrm{kg})\end{array}$ \\
\hline 125 & 156,23 \\
190 & 360,95 \\
260 & 675,91 \\
350 & 1224,83 \\
420 & 1763,76 \\
470 & 2208,70 \\
550 & 3024,59 \\
610 & 3720,49 \\
\hline
\end{tabular}

Assim, assumindo que a máxima vibração aceitável fosse de $15 \mathrm{~mm} / \mathrm{s}$, pode ser estabelecida a carga máxima por espera adequada para diferentes pontos no entorno dos pontos de detonação, de modo que não sejam causados danos a pessoas, estruturas e/ou equipamentos na vizinhança da operação.

\section{CONCLUSÕES}

Como salientado anteriormente, as vibrações e ruídos são os impactos que mais geram reclamações por parte da população vizinha a um empreendimento mineiro, cabendo aos órgãos ambientais fiscalizarem e regularem as atividades de desmonte de rochas com explosivos, forçando as empresas tomarem as medidas necessárias de forma a prever e atenuar estes efeitos. Diante disso, estabeleceuse uma campanha de monitoramento de vibrações e ruídos, onde por meio dos dados obtidos e da análise dos mesmos, puderam ser testados modelos de previsão que oferecessem um maior grau de confiança, obtendo assim uma equação de atenuação para um intervalo de confiança estipulado. 
Além disso, pode-se fazer um zoneamento de cargas máximas por espera na área de estudo, a partir do modelo de previsão determinado, na qual se pode constatar que quanto maior a distância a partir do local de detonação, menores devem ser as cargas máximas por espera, podendo aferir nos níveis de vibração gerados.

Durante o levantamento, pode-se constatar a ocorrência de valores baixos de níveis de vibração obtidos, possivelmente devido à grande atenuação das ondas em função da complexidade geológica com diversidade de intrusões e dobramentos, além do alto grau de fraturamento do maciço. Apenas um evento excedeu velocidade de pico de $10 \mathrm{~mm} / \mathrm{s}$, mas mesmo assim, este valor ficou abaixo do limite máximo estabelecido pela norma. No entanto, deve-se ressaltar a importância da utilização de modelos de atenuação baseados em intervalos de confiança, em função da grande dispersão dos valores, já que esta prática permite maior segurança na definição de cargas máximas, principalmente nos casos em que existam estruturas próximas.

Quantos aos dados de ruídos (sobrepressão acústica) pode-se observar que a maior parte dos níveis foram constantes, em um intervalo entre 120 e $140 \mathrm{~dB}$. Este fato pode ter ocorrido por fatores como a utilização de material inadequado como tampão nos furos ou mesmo comprimento inadequado deste, utilização de iniciação lateral com cordel detonante, sequência de iniciação ou tempos de retardo muito curtos e utilização de cordel exposto em superfície, na ligação dos furos. Cada uma destas possibilidades deve ser corrigida e verificada a eficácia da sua minimização com novas campanhas de monitoramento.

\section{REFERÊNCIAS}

ABNT. NBR-9653 - Guia para avaliação dos efeitos provocados pelo uso de explosivos nas minerações em áreas urbanas. Rio de Janeiro: 12 p., 2005.

CPRM - Companhia de Pesquisa de Recursos Minerais. Ministério de Minas e Energia. Programa Levantamentos Geológicos Básicos do Brasil. Cachoeira do Sul, Folha SH.22-Y-A. In: PORCHER, C. A.; LOPES, R. C. (Org.). Estado do Rio de Grande do Sul. Brasília, 2000.

BORTOLOTTO, O. J. Petrografia dos Mármores de Caçapava do Sul. Ciência e Natura: v. 9, p. 37-65, 1987.

DALLORA NETO, C. \& FERREIRA, G.C. Controle de vibrações geradas por desmonte de rocha com explosivos. Estudo de caso: calcário Cruzeiro, Limeira (SP). São Paulo: UNESP, Geociências, v. 25, n. 4, p. 455-466, 2006.

GAMA, C.D. Vibrações na atmosfera e nos terrenos adjacentes pós-detonação de explosivos - quantificação da sua afetação ambiental. Acústica 2008, Coimbra, Portugal: Universidade de Coimbra, 2008.

GIRAUDI, A.; CARDU, M. \& KECOJEVIC, V. An assessment of blasting vibrations: a case study on quarry operation. American Journal of Environmental Sciences, v. 5, n. 4, p. 467-473, 2009.

GOKHALE, B.V. Rotary drilling and blasting in large surface mines. Leiden, The Netherlands: CCR Press / Balkema, 777 p., 2011.

KONYA, C.J. Blast design. Montville, Ohio, USA: Intercontinental Development Corporation, 246 p., 1995.

KOPPE, J.C.; SALVADORETTI, P.; MUNARETTI, E. \& COSTA, J.F.C.L. Monitoramento geofísico de desmonte de rocha com utilização de explosivos em condições de risco elevado em zona urbana. Ouro Preto: REM - Revista Escola de Minas, 2001.

LARSON, R. \& FARBER, B. Estatística aplicada. 4. ed. São Paulo: Pearson Prentice Hall, 637 p., 2010.

MUNARETTI, E.; KOPPE, J.C.; MEDEIROS, M.A. \& COSTA, J.F.C.L. Proposta de delimitação de zona especial de mineração por meio de estudo de atenuação de onda sísmica gerada por desmonte com explosivos 
em Caxias do Sul, RS. IBRAM, 2006a.

MUNARETTI, E.; KOPPE, J.C.; SCHNACK, P. \& COSTA, J.F.C.L. Estudo da atenuação de onda sísmica para construção de depósito de resíduos industriais na Pedreira Expopedras, Carlos Barbosa, RS. IBRAM, $2006 b$.

OLOFSSON, S.O. Applied Explosives Technology for Construction and Mining. Sweden: APPLEX, 1994.

PERSSON, P.A.; HOLMBERG, R. \& LEE, J. Rock Blasting and Explosives Engineering. Boca Raton, Florida: CCR Press, 531 p., 1994.

SEN, G. Blasting Technology - For Mining and Civil Engineers. Sydney, Austrália: University of New South Wales Press LTD., 154 p., 1995.

SISKIND, D. \& STAGG, M.S. Blast Vibration Measurements Near and On Structure Foundations. USA: Report of investigations 8969, USBM - United States Bureau of Mines, 1985. 